\title{
Editorial
}

\section{The killer on road: Are we doing enough?}

\author{
U. Senarath ${ }^{1}, \mathrm{~S}$. Nandasena ${ }^{2}$
}

Road Traffic Accidents (RTA) have become a major killer causing a national tragedy in Sri Lanka. According to the Police reports, road deaths reached a record high of 2,800 in 2015 (1). That means, on average 8 persons die each day due to RTA. This is about 25 times higher than the number of maternal deaths reported in a year in Sri Lanka. The majority of the deceased were motor cyclists and pedestrians, while substantial numbers were among three-wheeler passengers and cyclists. A great majority of the victims was in their middle or early ages. Despite efforts taken by the successive Governments, the number of RTA has been rising rapidly in the recent past. During the last 10 years, more than 23,000 people have been killed in our roads, with more than 50,000 sustaining serious injuries. According to the annual health bulletin, traumatic injuries ranked the major cause of hospitalization over the last decade, and the number of admissions to state hospitals due to trauma was approximately 870,000 in 2013 (2).

Sri Lanka is proud of its achievements in health indicators, especially the impressive reductions in maternal and infant mortality in the last few decades. Sri Lankan health system, with a strong preventive arm and a well-integrated patient care service, is considered as a "model" in the developing world. However, lack of an effective preventive programme to combat RTA is a lacuna in this system. The rising number of victims of RTA will overburden the system compromising the quality of patient care in the hospitals, and also result in a disabled population in the community. With these challenges, will Sri Lanka be able to maintain its status of being the model health care delivery system in the developing world?

Having a safe road infrastructure and an efficient transport system is seen as a distinctive feature in a developed nation. If people die due to a preventable cause like road traffic accidents in an unacceptably high level, it is a reflection of a failure in the socio-political system. This will impede the development process in the long run. The impact on the health budget for management of the injured would be very high, while losses in the earnings will have a huge impact on family and national economy. It will have negative impact on tourism and related industries as well in future.

It is important to explore why such a high number of accidents occur in Sri Lanka. The main reason is due the reckless drivers and their non-compliance with road traffic regulations. Poor road conditions and failure by the authorities to strictly enforce the law have aggravated the situation. Another reason is the pedestrians' negligence. Incorrect designing of roads and infrastructure, poor condition of the vehicles, and increasing number of vehicles have also contributed to the even increasing number of accidents. However the core issue is lack of a sustainable national programme to ensure road safety.

Do we consider RTA a priority issue in our national agenda in relation to global context? The countries across the world including Sri Lanka adopted the sustainable development goals of the United Nations in 2015. 'Ensuring healthy lives and promoting well-being for all at all ages' is one the 17 sustainable development goals (Goal 3), under which a target was set to achieve a $50 \%$ reduction in the number of global deaths and injuries from road traffic accidents by 2030 (3). As a nation, are we ready to meet this challenge, or at least to march towards the target? 
Every citizen has the right to life in a civilized society. Should we consider every death or injury on the road as an 'accident' or 'misfortune' and not a crime? In Sri Lanka, ruthless drivers have killed so many innocent civilians on the road. Accidents of innocent children on a pedestrian crossing are reported in almost every other day. In some occasions the fault is totally the negligence by the pedestrian. Are the perpetrators penalized for the damage caused? If not what are the barriers that prevent appropriate action? Why such paradox in Sri Lanka, despite having a rich culture and religious diversity, the problem of RTA is becoming a severe threat to the lives? These questions are to be answered through a multifaceted anthropological approach.

RTA are preventable, and most of us know what to do to prevent RTA and ensure road safety, but there is a gap as how to do this uphill task. It evident that isolated or ad-hoc solutions will not help combat the issue, but a sustainable and strong solution is needed. It is imperative to have a great political will and commitment. The Sri Lanka Police and the National Council for Road Safety have taken several steps to minimize road accidents, such as improving road safety measures and educating the public regarding traffic rules. A conference on national road safety was held few years ago by the Transport Ministry and the National Council for Road Safety in collaboration with the United Nations and the World Health Organization. The Report of the Parliamentary Select Committee on Road Safety was presented at this meeting. Special attention has been given by the Directorate of Non-Communicable Diseases in the Ministry of Health to prevent and control RTA. Though these initiatives are admirable, the magnitude of the problem is so vast that their actions may not be sufficient to counteract the burden of RTA. Multi-sectoral effort is needed to address the issue of RTA. Almost all sectors have a role to play in addressing the issue of RTA. At least 3 major professionals namely public health, engineering and legal professionals should come forward to combat the issue of RTA. Above all, this is an area where the political leadership and commitment are greatly required. It is the responsibility of health professionals in this country to raise a stronger voice towards this cause.

\section{References}

1. Abeygoonewardena CR. Road safety - a matter of life and death. Sunday Times 28 Feb 2016. Web. 16 Mar 2016.

2. Medical Statistics Unit, Ministry of Health. Annual Health Bulletin 2013 - Sri Lanka. Colombo: Ministry of Health, Nutrition and Indigenous Medicine; 2015.

3. United Nations. Sustainable Development Goals. 2015 [Cited 16 March 2016]. Available from: http://www.un.org/sustainabledevelopment/health/.

\footnotetext{
1. Editor, Journal of the College of Community Physicians of Sri Lanka. Email: upul@commed.cmb.ac.lk

2. Co-Editor, Journal of the College of Community Physicians of Sri Lanka.

Email: sumalnandasena@gmail.com

Correspondence:

Email: editorjccpsl@gmail.com

Office: The College of Community Physicians of Sri Lanka,

No. 06, "Wijerama House", Wijerama Mawatha, Colombo 07, Sri Lanka

DOI: $10.4038 /$ jccpsl.v21i1.8079
} 